\title{
The effect of different micro-osteoperforation depths on the rate of orthodontic tooth movement: A single- center, single-blind, randomized clinical trial
}

\author{
Tugba Haliloglu Ozkan ${ }^{\mathrm{a}}$ (1) \\ Selim Arici ${ }^{b}$
}

${ }^{a}$ Department of Oral and Dental Health Program, Üsküdar University Vocational School of Health Services, İstanbul, Turkey

${ }^{b}$ Department of Orthodontics, Faculty of Dentistry, Ondokuz Mayıs University, Atakum, Samsun, Turkey

\begin{abstract}
Objective: This study aimed to identify the clinical effectiveness of two different penetration depths of micro-osteoperforations (MOPs) on the rate of orthodontic tooth movement. Methods: Twenty-four patients requiring the removal of the upper first premolar teeth were selected and randomly divided into two groups. The control group participants did not undergo MOPs. Participants in the experimental group underwent three MOPs each at 4-mm (MOP-4) and 7-mm (MOP-7) depths, which were randomly and equally performed to either the left or right side distal to the canine. The retraction amount was measured on three-dimensional digital models on the 28th day of retraction. MOP-related pain was measured using a visual analog scale (VAS). Between-group statistical differences in the VAS scores were determined using an independent $t$-test and those in canine retraction were determined using analysis of variance and posthoc Tukey test. Results: No significant difference was found between the MOP$4(1.22 \pm 0.29 \mathrm{~mm} / \mathrm{month})$ and MOP-7 $(1.29 \pm 0.31 \mathrm{~mm} / \mathrm{month})$ groups in terms of the canine retraction rate. Moreover, both the groups demonstrated a significantly higher canine movement than the control group $(0.88 \pm 0.19 \mathrm{~mm} /$ month). MOPs did not significantly affect the mesialization of the posterior teeth $(p>0.05)$. Moreover, the pain scores in the MOP-4 and MOP-7 groups were similar and showed no statistically significant difference. Conclusions: Three MOPs with a depth of $4 \mathrm{~mm}$ can be performed as an effective method to increase the rate of tooth movement. However, three MOPs with depths of 4-7 $\mathrm{mm}$ does not additionally enhance tooth movement.
\end{abstract}

[Korean J Orthod 2021;51(3):157-165]

Key words: Orthodontic miniscrew, Tooth movement

Received April 3, 2020; Revised November 25, 2020; Accepted December 7, 2020.

Corresponding author: Tugba Haliloglu Ozkan.

Lecturer, Department of Oral and Dental Health Program, Üsküdar University Vocational School of Health Services, Altunizade, Mahir iz Cd. No. 23, Üsküdar, İstanbul 34674, Turkey. Tel +90-505-598-80-28 e-mail dttuuba@gmail.com

How to cite this article: Haliloglu Ozkan T, Arici S. The effect of different microosteoperforation depths on the rate of orthodontic tooth movement: A single-center, single-blind, randomized clinical trial. Korean J Orthod 2021;51:157-165.

(C) 2021 The Korean Association of Orthodontists.

This is an Open Access article distributed under the terms of the Creative Commons Attribution Non-Commercial License (http://creativecommons.org/licenses/by-nc/4.0) which permits unrestricted non-commercial use, distribution, and reproduction in any medium, provided the original work is properly cited. 


\section{INTRODUCTION}

The duration of orthodontic treatment varies from several months to 3 years, with a mean treatment time of 19.9 months with fixed appliances. ${ }^{1}$ However, $74 \%$ of adolescent patients and $42 \%$ of adult patients expect a treatment time of less than 12 months. ${ }^{2}$ Considering that prolonged treatment induces adverse effects, such as root resorption, periodontal disease, decalcification, and temporomandibular dysfunction, shortening the treatment time seems to be of critical importance for both the clinician and patients. ${ }^{3}$

Although a wide variety of orthodontic treatment options exist today, from traditional ones (metal braces) to the newly developed alternatives (clear aligners and lingual braces), they all have the same drawback, i.e., the treatment time. To date, various interventions, including local injection of cellular mediators, ${ }^{4}$ physical-mechanical stimuli, ${ }^{5}$ and surgically assisted orthodontics, ${ }^{6}$ have been suggested to reduce the treatment time. It is now clearly understood that the crucial rate-limiting factor for accelerating tooth movement is an individual's biological bone response. Controlled microtrauma to the alveolar bone has been demonstrated to induce the regional acceleratory phenomenon (RAP), which leads to accelerated bone turnover. This in turn results in the increased secretion of naturally released inflammatory markers during orthodontic treatment and accelerated tooth movement. ${ }^{7}$ Recently, micro-osteoperforation (MOP) was suggested as a conservative, flapless surgical approach that uses the advantage of the RAP. The technique involves the placement of small transmucosal perforations within the alveolar bone. ${ }^{8}$ MOPs have been demonstrated to have clinical advantages such as decreased patient discomfort and obviates the need for raising flaps, suturing, and routine medication. ${ }^{9}$ Additionally, MOPs only require a couple of minutes of chairside time, and do not require any advanced training to perform, thereby allowing any clinician to perform it. $^{10}$

Although exploring a biological bone response seems exciting from a scientific perspective, there is a lack of evidence supporting such a new idea." The heterogeneity and relativity of the parameters (especially, the number, frequency, and depth of MOPs) tested in previous studies make it impossible to establish clear guidelines for the use of MOPs. Therefore, we designed a clinical study to gain a better understanding of the effectiveness of MOPs.

\section{Specific objectives and hypotheses}

The primary objective of this study was to clarify the clinical effectiveness of MOPs with different penetration depths. As the secondary objectives, the mesial movement of the molars and the MOP-related pain perception of patients were evaluated. The null hypothesis was that there would be a significant difference in the rate of tooth movement with increased depth of MOPs.

\section{MATERIALS AND METHODS}

\section{Trial design and changes after trial commencement}

This was a prospective, randomized clinical trial with two parallel arms, with a 1:1 allocation ratio, including a split-mouth design in the experimental arm. This randomized clinical trial followed the Consolidated Standards of Reporting Trials statement and guidelines. ${ }^{12}$ No design changes were made after trial commencement.

Table 1. Inclusion and exclusion criteria for patients

\begin{tabular}{ll}
\hline \multicolumn{1}{c}{ Inclusion criteria } & \multicolumn{1}{c}{ Exclusion criteria } \\
\hline Class I malocclusion & Class III malocclusion \\
Class II malocclusion (Dental, Division 1) & Sagittal and transversal skeletal problem \\
Malocclusion requiring the extraction of the upper first premolars & Aged $<16$ \\
Aged $>16$ & Cross bite \\
$\begin{array}{l}\text { Good general health } \\
\text { No medication }\end{array}$ & Abnormal chewing habit \\
$\begin{array}{l}\text { No radiographic or clinical evidence of bone loss } \\
\text { Good periodontal condition (probing depth 1-3 mm/ } \\
\text { pocket depth less than } 3 \mathrm{~mm} / \text { no bleeding while probing) }\end{array}$ & Previous orthodontic treatment \\
Healthy mental condition & Long term use of any drug \\
& Smoking \\
& Periodontal hygiene problem \\
\hline
\end{tabular}




\section{Participants, eligibility criteria, and settings}

This study was performed at a single center; the patients were recruited from the Orthodontic Department of Ondokuz Mayıs University from March 2017 to August 2017. This study was approved by the ethics committee of Ondokuz Mayıs University (No. 2017/69). Informed consent was obtained from all the participants included in the study. The inclusion and exclusion criteria for the study are summarized in Table 1 . The patient characteristics are presented in Table 2 .

\section{Interventions}

Twenty-four patients were randomly allocated to two groups: the control group without MOPs $(n=12)$ and the experimental group with MOPs $(n=12)$. In the experimental group, three MOPs each at 4-mm (MOP-4) and 7-mm (MOP-7) depths were randomly performed to either the left (MOP-4, $n=6$; MOP-7, $n=6$ ) or right side (MOP-4, $n=6$; MOP-7, $n=6$ ), distal to the canine.

In both the experimental and control groups, fixed appliances were placed after the extractions $(0.022 \times$ 0.028-inch [in] slot MBT prescription; Gemini, 3M Unitek, Monrovia, CA, USA). As extractions could induce RAP and, therefore, mimic the effect of MOPs, the teeth were leveled and aligned in the first 6 months. In this period, occlusal interferences during the canine retraction phase were also reduced. Leveling and aligning were done using the following MBT archwire sequence: 0.014-in, 0.016-in, and $0.019 \times 0.025$-in HANT (heatactivated $\mathrm{Ni}-\mathrm{Ti}$; 3M Unitek). To minimize binding and friction, the retraction phase was initiated after leaving

Table 2. Characteristics of patients in the experimental and control groups

\begin{tabular}{lccc}
\hline \multirow{2}{*}{ Group } & \multicolumn{2}{c}{ Sex } & \multirow{2}{*}{ Mean age } \\
\cline { 2 - 3 } & Female & Male & \\
\hline Experimental & 6 & 6 & $17.27 \pm 1.22$ \\
Control & 6 & 6 & $18.13 \pm 1.28$ \\
\hline
\end{tabular}

Values are presented as number only or mean \pm standard deviation.

Demographic statistics was performed. the $0.019 \times 0.025$-in stainless steel archwire passive for 4 weeks. Temporary anchorage devices (TADs) were placed between the roots of the second premolar and the first molar teeth to provide anchorage. To discriminate the pain caused by MOPs, the TADs were placed at the same time as the $0.019 \times 0.025$-in stainless steel archwire.

MOPs were performed under local anesthesia (2\% lidocaine with 1:100,000 epinephrine). A miniscrew (MTN-2; DesignMed, İstanbul, Turkey) with a diameter of $1.6 \mathrm{~mm}$ and a length of $8 \mathrm{~mm}$ was modified by placing 4 elastomeric ligatures (Leone, Firenze, ltaly) for MOP-4 and 1 elastomeric ligature for MOP-7 on the body of the miniscrew (Figure 1). The elastics were of medium size and had an inner diameter of $1.3 \mathrm{~mm}$. In the experimental group, three vertically lined, 4- and 7-mm deep MOPs were randomly performed to either the left or right side of the mouth, and the location was recorded. MOPs were created directly through the alveolar mucosa. To standardize the location of MOPs, a vertical line parallel to the long axis of the canine tooth was determined. The distance between the vertical line and the distal surface of the canine crown had to be $3 \mathrm{~mm}$. Thereafter, a probe was used to mark the location of the MOPs, with a screw width left between each perforation (Figure 2). The first MOP was performed as apically as possible to achieve more bodily movement. The second and third MOPs were located vertically toward the attached gingiva to follow the canine root. A constant force of $150 \mathrm{~g}$, as tested using a gauge, was applied to ligate the nitinol coil spring (Sentalloy; 3M Unitek) between the orthodontic miniscrew and the canine bracket hook. Following the intervention, only paracetamol administration was allowed for analgesia.

The patients in the MOP groups were asked to note their pain level separately for the right and left sides. The patients were asked to mark the highest level of pain felt within 24 hours on a visual analog scale (VAS). If the patients had to take analgesics during this period, they were asked to mark the level of pain they felt on both sides just before taking the drug. Moreover, only 1 patient scored the scale before the completion of 24 hours. The other patients did not require analgesics and had
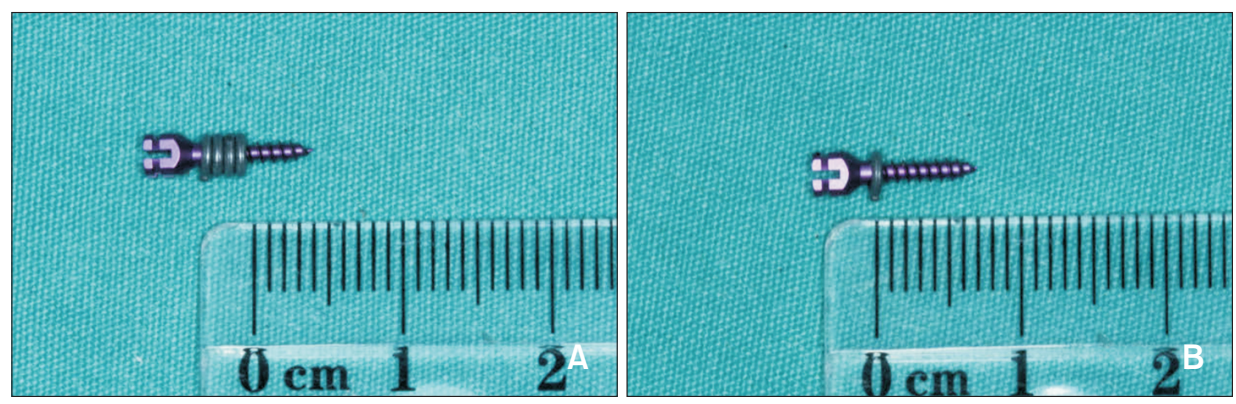

Figure 1. A, The screw set to 4-mm depth. B, The screw set to $7-\mathrm{mm}$ depth. 

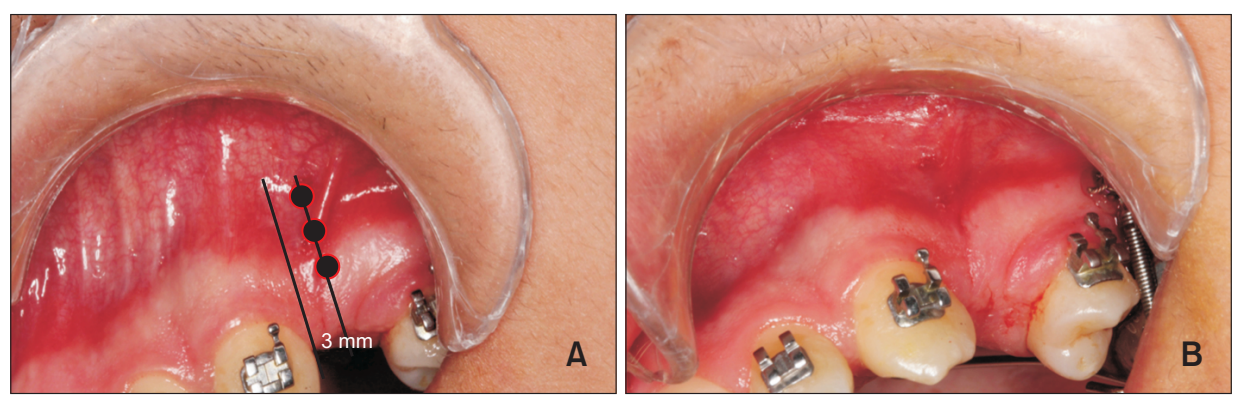

Figure 2. A, Schematic representation of the location of micro-osteoperforations (MOPs). B, The location after the application of MOPs.

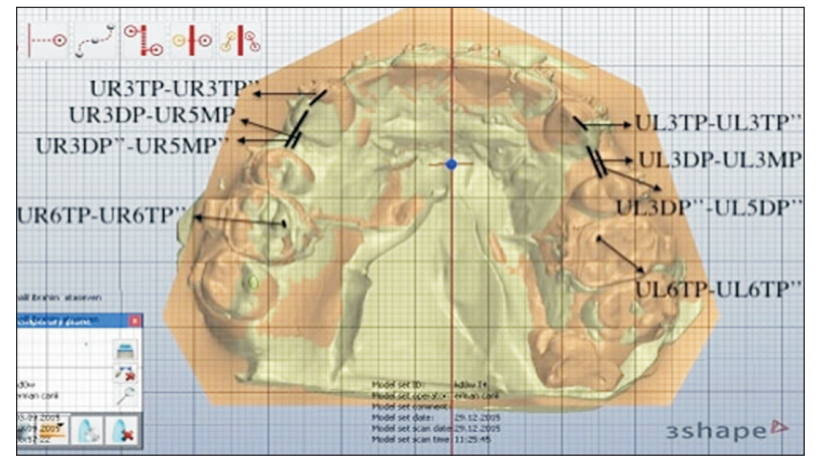

Figure 3. Three-dimensional measurements.

3, canine tooth; 5, second premolar tooth; 6 , first molar; UL, upper left; UR, upper right; TP, tubercule point; DP, distal point; MP, mesial point (" refers to the model obtained on the 28th day).

scored the highest level of pain they felt within 24 hours.

\section{Outcomes (primary and secondary) and changes after trial commencement}

The primary outcome was the monthly rate of canine retraction. Alginate impressions were taken before retraction and impression-taking was repeated at the 28th day of retraction when the study was concluded. Plaster models from the day the retraction started and from the 28th day were scanned and digitized using a software program (Orthoanalyzer; 3Shape, Copenhagen, Denmark) and superimposed. Superimposition was made on the third palatal rugae on the maxilla. The difference between the models at the start of retraction and the 28th day was specified by measuring the distance between the most distal point of the canine and the most mesial point of the second premolar from the occlusal view. Thus, canine movement was detected (Figure 3). Molar mesialization was measured using the mesiopalatal cusps of the first molar teeth. Intra-examiner reliability was determined by repeating each measurement twice at least 2 weeks apart. Random errors were calculated using the Dahlberg formula ${ }^{13}$ and were found to not exceed $\pm 0.1 \mathrm{~mm}$. The correlation coefficient in the reliability analysis (Cronbach's alpha) was 0.97 , indicating a high degree of similarity between the measurements. The VAS was used to measure the patients' highest level of pain felt within 24 hours.

\section{Sample size calculation}

Statistical power analysis was used to determine the number of samples at $\alpha=0.05$, at $80 \%$ power, and with a standard deviation of 0.34 . Sample size was calculated using the method described in a previous study. ${ }^{14}$ A 50\% difference in the rate of canine retraction, which was adopted to be clinically meaningful, was detected to calculate the sample size. Based on the calculation, 10 patients were found to be adequate for each arm. To compensate for follow-up loss, 12 patients were included in each group. Accordingly, 30 patients referred to the Orthodontic Department of Ondokuz Mayis University were examined. Of these, 4 patients did not meet the inclusion criteria and 2 declined to participate. Therefore, 24 patients were finally included in the study.

\section{Interim analyzes and stopping guidelines Not applicable.}

\section{Randomization}

At the beginning of the retraction period, a random allocation software (RAND function; Microsoft Excel 2010, Redmond, WA, USA) was used to allocate the patients into either the control (0) or the experimental group (1). Each patient was then asked to choose an opaque, brown envelope to determine whether the MOP-4 will be applied to the left or right side of the mouth. The investigator then applied MOP-7 to the other side of the patient's mouth. A secretary in our clinic was responsible for the randomization and monitoring of the process.

\section{Blinding}

The clinical procedures were managed by a single operator (T.O.). All the patients were blinded to the procedure as they had to fill the VAS for both the left and right sides of the mouth. However, measurements and analyses were performed by a different investigator (S.A.) who was blinded to all the procedures. 


\section{Statistical analysis}

SPSS for Windows, version 15.0 (SPSS Inc., Chicago, IL, USA) was used for all statistical analyses. The level of significance was set at $p<0.05$. Normality of data was checked using the Q-Q plot diagram and Shapiro-Wilk test (kurtosis and skewness values were also evaluated and found to be between 0 and \pm 1.5$).{ }^{15}$ All the variables were normally distributed. No significant difference was found between the male and female patients ( $p$ $>0.05)$. Hence, the data were pooled in terms of sex.

Both the left and right sides of the groups were used for the canine retraction measurements. Since the results of an independent $t$-test indicated no difference between the two sides in the control group, the right and left values were averaged for each patient and the data were analyzed as a single group $(n=12)$. Subsequently, analysis of variance (ANOVA) test was used to analyze the mean differences among the three groups (MOP-4/ MOP-7/control) in terms of canine retraction and molar mesialization. The results of ANOVA demonstrated a difference between the three groups; therefore, the posthoc Tukey test was performed to explore the significance.

The VAS scores were compared between the MOP-4 and MOP-7 groups. The difference in the VAS scores between the groups were evaluated using the independent $t$-test. All tests were performed at 95\% ( $p=0.05), 99 \%$ $(p=0.01)$, and $99.9 \%$ confidence intervals $(p=0.001)$.

\section{RESULTS}

\section{Participant flow}

A total of 24 patients were included in this study, and all of them completed the treatment. The study design and participant flow are illustrated in Figure 4.

\section{Baseline data}

Baseline characteristics were similar in the two groups.

Numbers analyzed for each outcome, estimation, and precision

All the results were evaluated considering three groups: MOP-4, MOP-7, and control. In 28 days, the mean amount of canine retraction was $1.22 \pm 0.29$ $\mathrm{mm}$ in the MOP-4 group, $1.29 \pm 0.31 \mathrm{~mm}$ in the MOP7 group, and $0.88 \pm 0.19 \mathrm{~mm}$ in the control group. Comparison of the effectiveness of the two penetration depths on canine retraction showed no clinically or statistically significant differences $(p>0.05)$. The MOP-4 and MOP-7 groups demonstrated a significantly higher canine retraction than did the control group $(p<0.05)$ (Table 3).

Molar mesialization was measured to be $0.17 \mathrm{~mm} /$ month in the MOP-4 group, $0.19 \mathrm{~mm} /$ month in the MOP-7 group, and $0.18 \mathrm{~mm} / \mathrm{month}$ in the control group. No significant differences were found between the three groups $(p>0.05)$. The mean VAS score was 42.75 (mild) in the MOP-4 group and 47.58 (moderate) in the MOP-7 group at the end of the 24 hours following the intervention. The results indicated no statistically significant difference (Table 4).

\section{Harms}

No miniscrew failure and no adverse effects, except moderate pain, were reported during and after the MOP procedure.

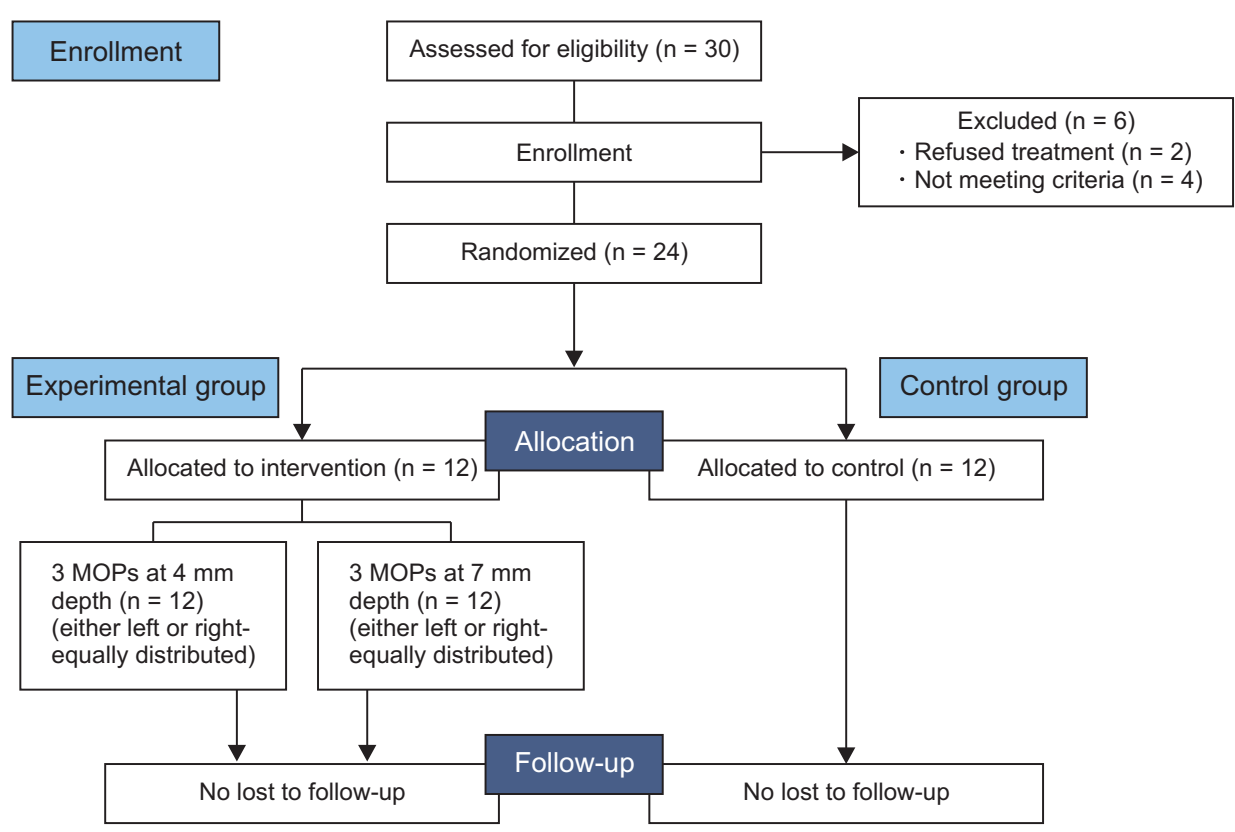

Figure 4. Flowchart of the events during the trial. MOPs, micro-osteoperforations. 
Table 3. Mean, standard deviation, and range of canine retraction in the experimental and control groups

\begin{tabular}{ccccccc}
\hline Group & N & Mean & Standard deviation & Minimum & Maximum & p-value \\
\hline MOP-4 $^{*}$ & 12 & 1.2200 & 0.29052 & 1.0356 & 1.4046 \\
MOP-7 $^{\dagger}$ & 12 & 1.2983 & 0.31214 & 1.1000 & 1.4967 & 0.016 \\
Control $^{* \dagger}$ & 12 & 0.8800 & 0.19904 & 0.7535 & 1.0065 & \\
\hline
\end{tabular}

ANOVA test was performed.

MOP, micro-osteoperforation; MOP-4, 4-mm MOP side; MOP-7, 7-mm MOP side.

The symbols $\left({ }^{*}+\right)$ indicate a statistically significant difference between the groups (Post-hoc Tukey test).

Table 4. Mean, standard deviation, and range of VAS scores

\begin{tabular}{ccccccc}
\hline Group & N & Mean & Standard deviation & Minimum & Maximum & p-value \\
\hline MOP-4 & 12 & 42.75 & 14.517 & 23.50 & 65.75 & 0.749 \\
MOP-7 & 12 & 47.25 & 16.638 & 24.75 & 76.50 & \\
\hline
\end{tabular}

Independent $t$-test was performed.

VAS, visual analog scale; MOP, micro-osteoperforation; MOP-4, 4-mm MOP side; MOP-7, 7-mm MOP side.

\section{DISCUSSION}

\section{Main findings in the context of the existing evidence and interpretation}

Evidence for the acceleration of tooth movement in conjunction with MOPs is conflicting. Some studies have demonstrated that MOP is a safe method ${ }^{8}$ that may facilitate root movement, ${ }^{16}$ induce cellular activity, and accelerate tooth movement ${ }^{8,17,18}$ without causing bacteremia. ${ }^{19}$ Flapless osteoperforations have been reported to increase the rate of orthodontic tooth movement toward the atrophic ridge, while maintaining a low bone densi$\mathrm{ty}^{20}$ and without an increased risk of root resorption. As a minimally invasive, flapless surgical intervention, MOP using miniscrews improve patient acceptance and reduce surgical risks. ${ }^{17}$ Conversely, some studies have demonstrated that MOPs do not have a significant effect on tooth movement and have limited and transitionary effects on the bone. ${ }^{21,22}$

The monthly rate of orthodontic tooth movement in patients treated using traditional methods involving continuous force application is $0.8-1.2 \mathrm{~mm} /$ month. $^{23}$ In a recently published meta-analysis, MOPs have been stated to increase the amount of canine retraction at the rate of $0.45 \mathrm{~mm} / \mathrm{month}$, and this change was found to be statistically significant. ${ }^{24}$ In our previously published study, we have reported that 3 MOPs with a $5-\mathrm{mm}$ depth could accelerate canine movement significantly at the rate of $1.76 \pm 0.66 \mathrm{~mm} / \mathrm{month}$, while the rate in the control group was $1.36 \pm 0.81 \mathrm{~mm} / \mathrm{month}$ (with a mean increase of $0.40 \mathrm{~mm} /$ month).$^{18} \mathrm{ln}$ the current study, the difference in the mean rate of canine retraction between the experimental $(1.25 \mathrm{~mm})$ and control groups $(0.88$ $\mathrm{mm}$ ) was $0.37 \mathrm{~mm}$, indicating a similarity with both the literature and our previous data.
A previous study reported a decrease in all the inflammatory markers tested, except interleukin-1, to the pre-retraction levels by the 28th day of MOP. ${ }^{9}$ Because of this reason, previous studies have presumably compared the effects of MOPs for 1 month., ${ }^{911,25}$ Additionally, in our previous study, considering the decreased effectiveness of the surgical insult by the fourth week of retraction (and, therefore, the level of cytokines and RAP response), we replaced the MOPs at the 28th day of retraction. ${ }^{18}$ However, the repeating MOPs at the end of the first 4 weeks did not enhance the retraction rate. Therefore, in the current study, we considered investigating the different depth effects of MOPs during the first 28 days of retraction.

We considered the type of movement as an important factor influencing the rate of movement. Therefore, we attempted to move the canine bodily by sliding the tooth on the $0.019 \times 0.025$-in stainless steel archwire on the $0.022 \times 0.028$-in slot. However, the force applied to the bracket hook was closer to the center of rotation than the force applied to the bracket slot; therefore, a more parallel movement was achieved.

In a recent study, the number of MOPs was kept constant in the two groups to investigate another variable of MOPs-the depth. Alikhani et al. ${ }^{7}$ tested the effect of 1,3 , and 4 MOPs on the rate of tooth movement and stated that 1 MOP was not effective to increase the rate of tooth movement, while 3 and 4 MOPs could be used to achieve an accelerated movement. Feizbakhsh et al. ${ }^{11}$ also reported that accelerated tooth movement could be achieved by performing only 2 MOPs. In the current study, our objective was primarily to investigate the effects of MOP depths; thus, we preferred to use 3 MOPs, which was the number used in the majority of previous studies. ${ }^{9,13,21,25,26}$ 
By increasing the magnitude of trauma, the magnitude of the inflammatory response can be increased. MOPs can be used to accomplish this response in two ways: (1) by increasing their number and (2) by increasing their depth of perforation. ${ }^{27}$ Considering the limited distance between the dental roots, a higher number of MOPs may not be suitable for every case. Therefore, it is more logical to increase the depth of MOPs. MOPs reportedly have both anabolic and catabolic effects. To achieve a catabolic effect (an effect that is mandatory while moving a tooth), penetration depths of 3-7 mm into the bone are recommended. ${ }^{7}$ For this purpose, we tested $7 \mathrm{~mm}$ as our upper limit of perforation. Since we have studied the MOP depth of $5 \mathrm{~mm}$ previously, we investigated whether even less trauma would accelerate tooth movement in this study; therefore, we set the lower depth limit as $4 \mathrm{~mm}$.

As a general rule does not exist about decision-making on the number and depth of MOPs, studies recommend considering the thickness of the soft tissue and cortical plate when applying the procedure. The thickness of the gingiva can be influenced by some factors such as genetic and racial factors, age, sex, dental arch location, and periodontal health. ${ }^{28,29}$ In the current study, as an inclusion criterion, we required all our patients to have a healthy periodontal condition without any radiographic evidence of bone loss. In a split-mouth design, age, sex, and genetic factors cannot be confounding factors. The dental arch location was identical, i.e., at the canine region of the mouth, as was the location of the MOPs, which was standard. Therefore, the effect of gingival thickness was minimized. However, as this factor can be relatively different in various study designs, further research involving the measurement of gingival thickness with a periodontal probe before the procedure is warranted.

Surgical techniques have been proposed to reduce the expected anchorage loss in the tooth by weakening the cortical bone around the canine and maintaining the integrity of the bone in the molar region. Thus, the spring used for retraction can work with the differential anchorage principle in the anterior and posterior regions. ${ }^{30}$ In the current study, TADs were used to preserve anchorage, and the molar teeth were not utilized as anchorage. This was aimed to examine whether the effect of the MOPs (and the RAP) extends to the posterior region by evaluating molar movement. MOPs have been shown to induce a biologically significant RAP effect farther away from the trauma sites. Significant decreases in cortical and trabecular bone density in the experimental bone were demonstrated 3-4 $\mathrm{mm}$ from the MOP sites, and dramatic tartrate resistant acid phosphatase activity after 2 weeks was observed $2.5 \mathrm{~mm}$ from the edges of the MOPs. ${ }^{31}$ In the current study, a slight mesial movement was observed at the molar teeth $(0.17 \mathrm{~mm}$ in the MOP4 group and $0.19 \mathrm{~mm}$ in the MOP-7 group). MOPs did not augment or inhibit molar mesialization either statistically or clinically probably because of the distance to the trauma site. Nevertheless, our results were consistent with those of previous reports assessing the effects of MOPs on molar movement., ${ }^{9,16,21}$

As expected, the examination of the VAS scores in the two MOP groups indicated that deeper (MOP-7) MOPs led to more pain than did the MOPs with a depth of 4 $\mathrm{mm}$. In the literature, various pain levels were reported to be associated with MOPs. Attri et al. ${ }^{26}$ reported no difference in pain perception with MOPs, while Aboalnaga et al. ${ }^{16}$ and Sivarajan et al. ${ }^{21}$ reported mild to severe pain.

\section{Generalizability}

In this study, the effects of MOPs on canine retraction were evaluated. These results, however, cannot be generalized to different approaches, like those for the elimination of crowding or tooth uprighting.

\section{Limitations}

A limitation of this study is that the TADs used could mimic the effect of MOPs; therefore, conventional anchorage control methods could be used instead of the posterior miniscrews used in this study to overcome this limitation.

\section{CONCLUSION}

- Three MOPs with a depth of 4-7 mm significantly accelerated canine retraction.

- As no difference was found between the MOP-4 and MOP-7 groups, the null hypothesis of this study was rejected.

- Future studies are warranted to investigate the effects of MOPs deeper than $7 \mathrm{~mm}$.

\section{CONFLICTS OF INTEREST}

No potential conflict of interest relevant to this article was reported.

\section{ACKNOWLEDGEMENTS}

We are grateful to Dr. Enes Özkan for editing the manuscript.

\section{REFERENCES}

1. Tsichlaki A, Chin SY, Pandis N, Fleming PS. How long does treatment with fixed orthodontic appliances last? A systematic review. Am J Orthod Den- 
tofacial Orthop 2016;149:308-18.

2. Uribe F, Padala S, Allareddy V, Nanda R. Patients', parents', and orthodontists' perceptions of the need for and costs of additional procedures to reduce treatment time. Am J Orthod Dentofacial Orthop 2014;145(4 Suppl):S65-73.

3. Talic NF. Adverse effects of orthodontic treatment: a clinical perspective. Saudi Dent J 2011;23:55-9.

4. Soma S, Matsumoto S, Higuchi Y, Takano-Yamamoto T, Yamashita K, Kurisu K, et al. Local and chronic application of PTH accelerates tooth movement in rats. J Dent Res 2000;79:1717-24.

5. Nishimura M, Chiba M, Ohashi T, Sato M, Shimizu $\mathrm{Y}$, lgarashi $\mathrm{K}$, et al. Periodontal tissue activation by vibration: intermittent stimulation by resonance vibration accelerates experimental tooth movement in rats. Am J Orthod Dentofacial Orthop 2008;133: 572-83.

6. Yavuz MC, Sunar 0, Buyuk SK, Kantarcı A. Comparison of piezocision and discision methods in orthodontic treatment. Prog Orthod 2018;19:44.

7. Alikhani M, Alansari S, Sangsuwon C, Alikhani M, Chou MY, Alyami B, et al. Micro-osteoperforations: minimally invasive accelerated tooth movement. Semin Orthod 2015;21:162-9.

8. Teixeira CC, Khoo E, Tran J, Chartres 1, Liu Y, Thant LM, et al. Cytokine expression and accelerated tooth movement. J Dent Res 2010;89:1135-41.

9. Alikhani M, Raptis M, Zoldan B, Sangsuwon C, Lee YB, Alyami B, et al. Effect of micro-osteoperforations on the rate of tooth movement. Am J Orthod Dentofacial Orthop 2013;144:639-48.

10. Nicozisis JL. Accelerated orthodontics through microosteoperforation. Orthod Pract 2013;4:56-7.

11. Feizbakhsh M, Zandian D, Heidarpour M, Farhad SZ, Fallahi HR. The use of micro-osteoperforation concept for accelerating differential tooth movement. J World Fed Orthod 2018;7:56-60.

12. Schulz KF, Altman DG, Moher D; CONSORT Group. CONSORT 2010 statement: updated guidelines for reporting parallel group randomised trials. BMC Med 2010;8:18.

13. Galvão MCS, Sato JR, Coelho EC. Dahlberg formula: a novel approach for its evaluation. Dental Press J Orthod 2012;17:115-24.

14. Alkebsi A, Al-Maaitah E, Al-Shorman H, Abu Alhaija E. Three-dimensional assessment of the effect of micro-osteoperforations on the rate of tooth movement during canine retraction in adults with Class 11 malocclusion: a randomized controlled clinical trial. Am J Orthod Dentofacial Orthop 2018;153:771-85.

15. George D, Mallery P. SPSS for Windows step by step: a simple guide and reference, 17.0 update. Boston: Pearson; 2010.
16. Aboalnaga AA, Salah Fayed MM, El-Ashmawi NA, Soliman SA. Effect of micro-osteoperforation on the rate of canine retraction: a split-mouth randomized controlled trial. Prog Orthod 2019;20:21.

17. Cheung T, Park J, Lee D, Kim C, Olson J, Javadi S, et al. Ability of mini-implant-facilitated micro-osteoperforations to accelerate tooth movement in rats. Am J Orthod Dentofacial Orthop 2016;150:958-67.

18. Haliloglu-Ozkan T, Arici N, Arici S. In-vivo effects of flapless osteopuncture-facilitated tooth movement in the maxilla and the mandible. J Clin Exp Dent 2018;10:e761-7.

19. Azeem M, Ul Haq A, llyas M, Ul Hamid W, Hayat $\mathrm{MB}$, Jamal F, et al. Bacteremia after micro-osteoperforation. Int Orthod 2018;16:463-9.

20. Lee JW, Cha JY, Park KH, Kang YG, Kim SJ. Effect of flapless osteoperforation-assisted tooth movement on atrophic alveolar ridge: histomorphometric and gene-enrichment analysis. Angle Orthod 2018; 88:82-90.

21. Sivarajan S, Doss JG, Papageorgiou SN, Cobourne MT, Wey MC. Mini-implant supported canine retraction with micro-osteoperforation: a split-mouth randomized clinical trial. Angle Orthod 2019;89:183-9.

22. Cramer CL, Campbell PM, Opperman LA, Tadlock $\mathrm{LP}$, Buschang PH. Effects of micro-osteoperforations on tooth movement and bone in the beagle maxilla. Am J Orthod Dentofacial Orthop 2019;155:681-92.

23. Andrade 1 Jr, Sousa AB, da Silva GG. New therapeutic modalities to modulate orthodontic tooth movement. Dental Press J Orthod 2014;19:123-33.

24. Shahabee M, Shafaee H, Abtahi M, Rangrazi A, Bardideh E. Effect of micro-osteoperforation on the rate of orthodontic tooth movement-a systematic review and a meta-analysis. Eur J Orthod 2020; 42:211-21.

25. Kundi 1, Alam MK, Shaheed S. Micro-osteo perforation effects as an intervention on canine retraction. Saudi Dent J 2020;32:15-20.

26. Attri S, Mittal R, Batra P, Sonar S, Sharma K, Raghavan $\mathrm{S}$, et al. Comparison of rate of tooth movement and pain perception during accelerated tooth movement associated with conventional fixed appliances with micro-osteoperforations- a randomised controlled trial. J Orthod 2018;45:225-33.

27. Alikhani M. Clinical guide to accelerated orthodontics with a focus on micro-osteoperforations. Cham: Springer; 2017.

28. Kolte R, Kolte A, Mahajan A. Assessment of gingival thickness with regards to age, gender and arch location. J Indian Soc Periodontol 2014;18:478-81.

29. Müller HP, Könönen E. Variance components of gingival thickness. J Periodontal Res 2005;40:23944. 
30. Işik Aslan B, Baloştuncer B, Dinçer M. Are there differences on tooth movement between different sectional canine retractors? J Orofac Orthop 2013;74: 226-35.

31. Kini U, Nandeesh BN. Physiology of bone forma- tion, remodeling, and metabolism. In: Fogelman I, Gnanasegaran G, van der Wall H, eds. Radionuclide and hybrid bone imaging. Berlin, Heidelberg: Springer; 2012. p. 29-57. 